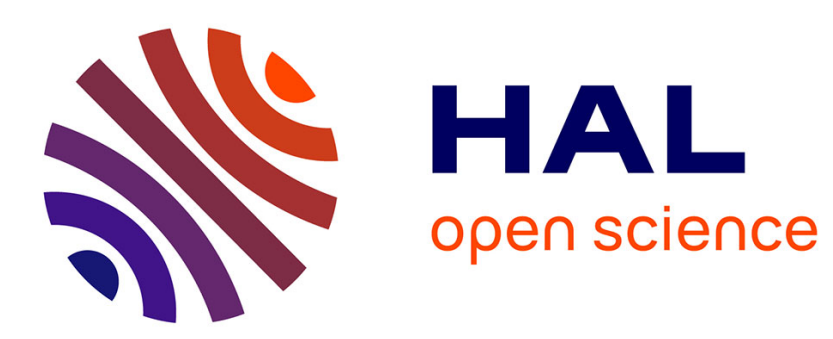

\title{
Semiclassical resolvent estimates for Holder potentials
} Georgi Vodev

\section{To cite this version:}

Georgi Vodev. Semiclassical resolvent estimates for Holder potentials. Pure and Applied Analysis, 2020, 2 (4), pp.841-860. hal-02492811v3

\section{HAL Id: hal-02492811 \\ https://hal.science/hal-02492811v3}

Submitted on 3 Aug 2020

HAL is a multi-disciplinary open access archive for the deposit and dissemination of scientific research documents, whether they are published or not. The documents may come from teaching and research institutions in France or abroad, or from public or private research centers.
L'archive ouverte pluridisciplinaire $\mathbf{H A L}$, est destinée au dépôt et à la diffusion de documents scientifiques de niveau recherche, publiés ou non, émanant des établissements d'enseignement et de recherche français ou étrangers, des laboratoires publics ou privés. 


\title{
SEMICLASSICAL RESOLVENT ESTIMATES FOR HÖLDER POTENTIALS
}

\author{
GEORGI VODEV
}

\begin{abstract}
We first prove semiclassical resolvent estimates for the Schrödinger operator in $\mathbb{R}^{d}$, $d \geq 3$, with real-valued potentials which are Hölder with respect to the radial variable. Then we extend these resolvent estimates to exterior domains in $\mathbb{R}^{d}, d \geq 2$, and real-valued potentials which are Hölder with respect to the space variable. As an application, we obtain the rate of the decay of the local energy of the solutions to the wave equation with a refraction index which may be Hölder, Lipschitz or just $L^{\infty}$.
\end{abstract}

Key words: Schrödinger operator, resolvent estimates, Hölder potentials.

\section{INTRODUCTION AND STATEMENT OF RESULTS}

In this paper we are going to study the resolvent of the Schrödinger operator

$$
P(h)=-h^{2} \Delta+V(x)
$$

where $0<h \leq 1$ is a semiclassical parameter, $\Delta$ is the negative Laplacian in $\mathbb{R}^{d}, d \geq 2$, and $V \in L^{\infty}\left(\mathbb{R}^{d}\right)$ is a real-valued potential satisfying the condition

$$
V(x) \leq p(|x|)
$$

where $p(r)>0, r \geq 0$, is a decreasing function such that $p(r) \rightarrow 0$ as $r \rightarrow \infty$. More precisely, we are interested in bounding the quantity

$$
g_{s}^{ \pm}(h, \varepsilon):=\log \left\|(|x|+1)^{-s}(P(h)-E \pm i \varepsilon)^{-1}(|x|+1)^{-s}\right\|_{L^{2} \rightarrow L^{2}}
$$

from above by an explicit function of $h$, independent of $\varepsilon$, without imposing extra assumptions on the function $p$. Here $L^{2}:=L^{2}\left(\mathbb{R}^{d}\right), 0<\varepsilon<1, s>1 / 2$ is independent of $h$ and $E>0$ is a fixed energy level independent of $h$. Instead, we impose some regularity on the potential with respect to the radial variable $r=|x|$. Note that througout this paper the space $C^{1}$ will denote the Lipschitz functions, that is, the ones with first derivatives belonging to $L^{\infty}$ (and not necessairily continuous).

We will first extend Datchev's result [5] to a larger class of potentials. Recall that in [5] the bound

$$
g_{s}^{ \pm}(h, \varepsilon) \leq C h^{-1}
$$

is proved when $d \geq 3$, with some constant $C>0$ independent of $h$ and $\varepsilon$, for potentials $V \in C^{1}\left(\overline{\mathbb{R}^{+}}\right)$with respect to the radial variable $r$ and satisfying $(1.1)$ with $p(|x|)=C_{1}(|x|+1)^{-\delta}$ as well as the condition

$$
\partial_{r} V(x) \leq C_{2}(|x|+1)^{-\beta}
$$

where $C_{1}, C_{2}, \delta>0$ and $\beta>1$ are some constants. We will prove the following

Theorem 1.1. Let $d \geq 3$ and suppose that the potential $V$ satisfies the conditions (1.1) and (1.3). Then there exists a constant $C>0$ independent of $h$ and $\varepsilon$ but depending on $s, E$ and the function $p$, such that the bound (1.2) holds for all $0<h \leq 1$. 
Note that the bound (1.2) was first proved for smooth potentials in [2]. A high-frequency analog of (1.2) on Riemannian manifolds was also proved in [1] and [3]. When $d=2$ the bound (1.2) is proved in [10] for potentials $V \in C^{1}\left(\mathbb{R}^{2}\right)$ satisfying (1.1) with $p(|x|)=C_{1}(|x|+1)^{-\delta}$ as well as the condition

$$
|\nabla V(x)| \leq C_{2}(|x|+1)^{-\beta}
$$

where $C_{1}, C_{2}, \delta>0$ and $\beta>1$ are some constants.

On the other hand, for compactly supported $L^{\infty}$ potentials without any regularity the following weaker bound

$$
g_{s}^{ \pm}(h, \varepsilon) \leq C h^{-4 / 3} \log \left(h^{-1}\right)
$$

was proved for $0<h \ll 1$ in [7] and [11] when $d \geq 2$. When $d \geq 3$ the bound (1.5) has been extended in $[12]$ to potentials satisfying the condition

$$
|V(x)| \leq C_{3}(|x|+1)^{-\delta}
$$

where $C_{3}>0$ and $\delta>3$ are some constants. Note that (1.5) has been recently proved in [4] for potentials satisfying (1.6) with $\delta>2$. For potentials satisfying (1.6) with $1<\delta \leq 3$ the much weaker bound

$$
g_{s}^{ \pm}(h, \varepsilon) \leq C h^{-\frac{2 \delta+5}{3(\delta-1)}}\left(\log \left(h^{-1}\right)\right)^{\frac{1}{\delta-1}}
$$

was proved in [13].

In the present paper we show that the bound (1.5) can be improved if some small regularity of the potential is assumed. To be more precise, given $0<\alpha<1$ and $\beta>0$, we introduce the space $C_{\beta}^{\alpha}\left(\overline{\mathbb{R}^{+}}\right)$of all Hölder functions $a$ such that

$$
\sup _{r^{\prime} \geq 0: 0<\left|r-r^{\prime}\right| \leq 1} \frac{\left|a(r)-a\left(r^{\prime}\right)\right|}{\left|r-r^{\prime}\right|^{\alpha}} \leq C(r+1)^{-\beta}, \quad \forall r \in \overline{\mathbb{R}^{+}},
$$

for some constant $C>0$. We now suppose that the function $V(r, w):=V(r w)$ satisfies the condition

$$
V(\cdot, w) \in C_{4}^{\alpha}\left(\overline{\mathbb{R}^{+}}\right), \quad 0<\alpha<1,
$$

uniformly in $w \in \mathbb{S}^{d-1}$. We have the following

Theorem 1.2. Let $d \geq 3$ and suppose that the potential $V$ satisfies the conditions (1.1) and (1.8). Then there exists a constant $C>0$ independent of $h$ and $\varepsilon$ but depending on $s, E$ and the function $p$, such that the bound

$$
g_{s}^{ \pm}(h, \varepsilon) \leq C h^{-4 /(\alpha+3)} \log \left(h^{-1}\right)+C
$$

holds for all $0<h \leq 1$.

The proof of the above theorems is based on the global Carleman estimates proved in [13] but with different phase and weight functions (see Theorem 4.1). In fact, in the case of Hölder or Lipschitz potentials we need to construct better phase functions and hence get better Carleman estimates. Such functions are constructed in Section 2 modifying the construction in [13] in a suitable way. In order that the Carleman estimates (see (4.1) and (4.6) below) hold, the phase and weight functions must satisfy some inequalities (see (2.5), (2.9) and (2.21) below), so most of the proof of the above theorems consists of proving these inequalities. Note also that the above theorems have been recently proved in [4] by using similar Carleman estimates but with a better choice of the phase function. Consequently, the bound (1.9) is proved in [4] for a larger 
class of $\alpha$-Hölder potentials. On the other hand, it is shown in [15] that the logarithmic term in the right-hand side of (1.9) can be removed for radial potentials.

We next extend the above results to arbitrary obstacles and all dimensions $d \geq 2$. To do so, we need to replace the conditions (1.3) and (1.8) by stronger ones. To be more precise, we let $\Omega \subset \mathbb{R}^{d}, d \geq 2$, be a connected domain with smooth boundary $\partial \Omega$ such that $\mathbb{R}^{d} \backslash \Omega$ is compact. Let $r_{0}>0$ be such that $\mathbb{R}^{d} \backslash \Omega \subset\left\{x \in \mathbb{R}^{d}:|x| \leq r_{0}\right\}$. Given a real-valued potential $V \in L^{\infty}(\Omega)$ satisfying (1.1) for $|x| \geq r_{0}$, we denote by $P(h)$ the Dirichlet self-adjoint realisation of the operator $-h^{2} \Delta+V(x)$ on the Hilbert space $L^{2}(\Omega)$. We define the quantity $g_{s}^{ \pm}$in the same way as above with $L^{2}=L^{2}(\Omega)$. Given $0<\alpha \leq 1$ and $\beta>0$, we introduce the space $C_{\beta}^{\alpha}(\bar{\Omega})$ of all Hölder functions $a$ such that

$$
\sup _{x^{\prime} \in \bar{\Omega}: 0<\left|x-x^{\prime}\right| \leq 1} \frac{\left|a(x)-a\left(x^{\prime}\right)\right|}{\left|x-x^{\prime}\right|^{\alpha}} \leq C(|x|+1)^{-\beta}, \quad \forall x \in \bar{\Omega},
$$

for some constant $C>0$. Note that the case $\alpha=1$ corresponds to the Lipschitz functions. We suppose that

$$
V \in C_{\beta}^{\alpha}(\bar{\Omega}), \quad 0<\alpha \leq 1, \beta>1 .
$$

We have the following

Theorem 1.3. Let $d \geq 2$ and suppose that the potential $V \in L^{\infty}(\Omega)$ satisfies (1.1) for $|x| \geq r_{0}$. If $V$ satisfies (1.10) with $\alpha=1$ and $\beta>1$, then the bound (1.2) holds for all $0<h \leq 1$. If $V$ satisfies (1.10) with $0<\alpha<1$ and $\beta=4$, then the bound (1.9) holds for all $0<h \leq 1$.

To prove this theorem we follow the same strategy as in [14], where the bound (1.5) is proved in all dimensions $d \geq 2$ for potentials $V \in L^{\infty}(\Omega)$ satisfying (1.6). It consists of gluing up two different types of estimates - one in a compact set coming from the local Carleman estimates proved in [8] (see Theorem 3.1) with a global Carleman estimate outside a sufficently big compact (see Theorem 4.2). This is carried out in Section 4.

Theorem 1.3 together with Theorem 1.1 of [14] allow us to get uniform bounds for the resolvent of the Dirichlet self-adjoint realisation, $G$, of the operator $-n(x)^{-1} \Delta$ in the Hilbert space $H=$ $L^{2}(\Omega, n(x) d x)$, where $n \in L^{\infty}(\Omega)$ is a real-valued function called refraction index satisfying the conditions

$$
n_{1} \leq n(x) \leq n_{2} \quad \text { in } \quad \Omega,
$$

with some constants $n_{1}, n_{2}>0$, and

$$
|n(x)-1| \leq C(|x|+1)^{-\delta} \quad \text { in } \Omega,
$$

with some constants $C, \delta>0$. More precisely, we have the following

Corollary 1.4. Suppose that the function $n$ satisfies the conditions (1.11) and (1.12). Then, given any $s>1 / 2$ and $\lambda_{0}>0$ there is a constant $C>0$ depending on $s$ and $\lambda_{0}$ such that the estimate

$$
\left\|(|x|+1)^{-s}\left(G-\lambda^{2} \pm i \varepsilon\right)^{-1}(|x|+1)^{-s}\right\|_{H \rightarrow H} \leq e^{C \psi(\lambda)}
$$

holds for all $\lambda \geq \lambda_{0}$ uniformly in $\varepsilon$, where $\psi(\lambda)=\lambda^{4 / 3} \log (\lambda+1)$ if $n \in L^{\infty}(\Omega)$ satisfies (1.12) with $\delta>3, \psi(\lambda)=\lambda^{4 /(\alpha+3)} \log (\lambda+1)$ if $n \in C_{4}^{\alpha}(\bar{\Omega})$ with $0<\alpha<1, \psi(\lambda)=\lambda$ if $n \in C_{\beta}^{1}(\bar{\Omega})$ with $\beta>1$.

To get (1.13) we apply the theorems mentioned above with $h=\lambda_{0} / \lambda, V=\lambda_{0}^{2}(1-n), E=\lambda_{0}^{2}$ and $\varepsilon$ replaced by $\varepsilon h^{2} n$. 
Using Corollary 1.4 one can extend Shapiro's result [9] on the local energy decay of the solutions of the following wave equation

$$
\left\{\begin{array}{l}
\left(n(x) \partial_{t}^{2}-\Delta\right) u(t, x)=0 \quad \text { in } \mathbb{R} \times \Omega, \\
u(t, x)=0 \text { on } \mathbb{R} \times \partial \Omega, \\
u(0, x)=f_{1}(x), \partial_{t} u(0, x)=f_{2}(x) \text { in } \Omega .
\end{array}\right.
$$

Given any $r_{0} \gg 1$, denote $\Omega_{r_{0}}=\left\{x \in \Omega:|x| \leq r_{0}\right\}$. We have the following

Corollary 1.5. Suppose that the function $n$ satisfies (1.11) and that $n=1$ outside some compact subset of $\Omega$. Then, the solution $u(t, x)$ to the equation (1.14) with compactly supported initial data $\left(f_{1}, f_{2}\right) \in H_{0}^{2}(\Omega) \times H_{0}^{1}(\Omega)$ satisfies the estimate

$$
\|\nabla u(t, \cdot)\|_{L^{2}\left(\Omega_{r_{0}}\right)}+\left\|\partial_{t} u(t, \cdot)\right\|_{L^{2}\left(\Omega_{r_{0}}\right)} \leq C \omega(t)\left(\left\|f_{1}\right\|_{H^{2}(\Omega)}+\left\|f_{2}\right\|_{H^{1}(\Omega)}\right)
$$

for $t \gg 1$, where

$$
\omega(t)=\left(\frac{\log \log t}{\log t}\right)^{3 / 4}
$$

Suppose in addition that $n \in C^{\alpha}(\bar{\Omega})$ with $0<\alpha \leq 1$. Then the estimate (1.15) holds with

$$
\omega(t)=\left(\frac{\log \log t}{\log t}\right)^{(\alpha+3) / 4}
$$

if $0<\alpha<1$, and with $\omega(t)=(\log t)^{-1}$ if $\alpha=1$. The estimate (1.15) remains valid when $\Omega=\mathbb{R}^{d}$.

Remark 1. In view of the recent results in [15], when $\Omega=\mathbb{R}^{d}, d \geq 3$ and the function $n$ depends only on the radial variable $r$, the estimate (1.15) holds with $\omega(t)=(\log t)^{-3 / 4}$ if $n \in L^{\infty}$, and with $\omega(t)=(\log t)^{-(\alpha+3) / 4}$ if $n$ is $\alpha$ - Hölder in $r$.

Note that estimates similar to (1.15) were first proved by Burq [1] in the case $n \equiv 1$. Note also that an analog of the above theorem is proved by Shapiro [9] in the case $\Omega=\mathbb{R}^{d}$. Then an estimate similar to (1.15) is proved with $\omega(t)$ replaced by $(\log t)^{-3 / 4+\epsilon}, \epsilon>0$ being arbitrary. Moreover, if in addition the function $n$ is supposed Lipschitz, then the decay rate is improved to $\omega(t)=(\log t)^{-1}$. The proof in [9] is based on the resolvent estimates obtained in [5], [10] and $[11]$.

The assumption that $n=1$ outside some compact is only necessary to study the low-frequency behavior of the cut-off resolvent of $G$. Indeed, under this assumption one can easily see that this behavior is exactly the same as in the case when $n \equiv 1$, which in turn is well-known (e.g. see Appendix B.2 of [1]). Therefore, in this case the low-frequency analysis can be carried out in precisely the same way as in [9]. Most probably, the condition (1.12) with $\delta>2$ would be enough. The high-frequency analysis in our case is also very similar to that one in [9] with some slight modifications allowing to deduce from (1.13) the sharp decay rate $\omega(t)$ (instead of $\left.(\log t)^{-3 / 4+\epsilon}\right)$.

\section{Construction of the phase And Weight Functions}

Let $\rho \in C_{0}^{\infty}([0,1]), \rho \geq 0$, be a real-valued function independent of $h$ such that $\int_{0}^{\infty} \rho(\sigma) d \sigma=1$. If $V$ satisfies (1.8), we approximate it by the function

$$
V_{\theta}(r, w)=\theta^{-1} \int_{0}^{\infty} \rho\left(\left(r^{\prime}-r\right) / \theta\right) V\left(r^{\prime}, w\right) d r^{\prime}=\int_{0}^{\infty} \rho(\sigma) V(r+\theta \sigma, w) d \sigma
$$


where $\theta=h^{2 /(\alpha+3)}$. Indeed, we have

$$
\begin{gathered}
\left|V(r, w)-V_{\theta}(r, w)\right| \leq \int_{0}^{\infty} \rho(\sigma)|V(r+\theta \sigma, w)-V(r, w)| d \sigma \\
\lesssim \theta^{\alpha}(r+1)^{-4} \int_{0}^{\infty} \sigma^{\alpha} \rho(\sigma) d \sigma \lesssim \theta^{\alpha}(r+1)^{-4} .
\end{gathered}
$$

This bound together with (1.1) imply

$$
V_{\theta}(r, w) \leq p(r)+\mathcal{O}\left((r+1)^{-4}\right) .
$$

Clearly, $V_{\theta}$ is $C^{1}$ with respect to the variable $r$ and its first derivative $V_{\theta}^{\prime}$ is given by

$$
\begin{gathered}
V_{\theta}^{\prime}(r, w)=\theta^{-2} \int_{0}^{\infty} \rho^{\prime}\left(\left(r^{\prime}-r\right) / \theta\right) V\left(r^{\prime}, w\right) d r^{\prime} \\
=\theta^{-1} \int_{0}^{\infty} \rho^{\prime}(\sigma) V(r+\theta \sigma, w) d \sigma=\theta^{-1} \int_{0}^{\infty} \rho^{\prime}(\sigma)(V(r+\theta \sigma, w)-V(r, w)) d \sigma
\end{gathered}
$$

where we have used that $\int_{0}^{\infty} \rho^{\prime}(\sigma) d \sigma=0$. Hence

$$
\left|V_{\theta}^{\prime}(r, w)\right| \lesssim \theta^{-1+\alpha}(r+1)^{-4} \int_{0}^{\infty} \sigma^{\alpha}\left|\rho^{\prime}(\sigma)\right| d \sigma \lesssim \theta^{-1+\alpha}(r+1)^{-4} .
$$

We now construct the weight function $\mu$ as follows:

$$
\mu(r)= \begin{cases}(r+1)^{2 k}-(r+1)^{2 k_{0}} & \text { for } 0 \leq r \leq a, \\ (a+1)^{2 k}-(a+1)^{2 k_{0}}+(a+1)^{-2 s+1}-(r+1)^{-2 s+1} & \text { for } r \geq a,\end{cases}
$$

where $a=a_{0} h^{-m}$ with $a_{0} \gg 1$ independent of $h, m=0$ if $V$ satisfies (1.3) and $m=2$ if $V$ satisfies (1.8). We choose $k=\frac{1}{4} \min \{1, \beta-1\}, k_{0}=0$ if $V$ satisfies (1.3), and $k=1, k_{0}=1 / 2$ if $V$ satisfies (1.8). Furthermore, $s$ is independent of $h$ such that

$$
\frac{1}{2}<s< \begin{cases}\frac{1}{4} \min \{3, \beta+1\} & \text { if } V \text { satisfies (1.3) } \\ \frac{3}{4} & \text { if } V \text { satisfies (1.8). }\end{cases}
$$

Clearly, the first derivative of $\mu$ is given by

$$
\mu^{\prime}(r)= \begin{cases}2 k(r+1)^{2 k-1}-2 k_{0}(r+1)^{2 k_{0}-1} & \text { for } 0 \leq r<a \\ (2 s-1)(r+1)^{-2 s} & \text { for } r>a\end{cases}
$$

We have the following

Lemma 2.1. For all $r>0, r \neq a$, we have the inequalities

$$
\begin{aligned}
& 2 r^{-1} \mu(r)-\mu^{\prime}(r) \geq 0, \\
& \frac{\mu(r)^{j}}{\mu^{\prime}(r)} \lesssim a^{2 k j}(r+1)^{2 s},
\end{aligned}
$$

for every $j \geq 0$.

Proof. It is shown in Section 2 of [13] that when $k_{0}=0$ the inequality (2.5) holds for all $0<k \leq 1$. Here we will prove it when $\nu:=2 k-2 k_{0} \geq 1$ and $0<k \leq 1$. For $r<a$ we have

$$
\begin{gathered}
2 \mu(r)-r \mu^{\prime}(r) \\
=2(1-k)(r+1)^{2 k}-2\left(1-k_{0}\right)(r+1)^{2 k_{0}}+2 k(r+1)^{2 k-1}-2 k_{0}(r+1)^{2 k_{0}-1} \\
=2(r+1)^{2 k_{0}-1}\left((1-k)(r+1)^{\nu+1}-\left(1-k_{0}\right)(r+1)+k(r+1)^{\nu}-k_{0}\right)
\end{gathered}
$$




$$
\begin{gathered}
=2(r+1)^{2 k_{0}-1}\left((1-k) r\left((r+1)^{\nu}-1\right)+(r+1)^{\nu}-\nu r / 2-1\right) \\
\geq 2(r+1)^{2 k_{0}-1}\left((r+1)^{\nu}-\nu r / 2-1\right) \geq \nu r(r+1)^{2 k_{0}-1}>0
\end{gathered}
$$

where we have used the well-known inequality

$$
(r+1)^{\nu} \geq \nu r+1
$$

as long as $\nu \geq 1$. For $r>a$ the left-hand side of (2.5) is bounded from below by

$$
2 r^{-1}\left((a+1)^{2 k}-(a+1)^{2 k_{0}}-s\right)>0
$$

provided $a$ is taken large enough. To prove (2.6) observe that for $r<a$ we have

$$
\mu^{\prime}(r) \geq 2\left(k-k_{0}\right)(r+1)^{2 k-1} \geq 2\left(k-k_{0}\right)(r+1)^{-1} \geq 2\left(k-k_{0}\right)(r+1)^{-2 s}
$$

which clearly implies the bound (2.6) with $j=0$. This together with the fact that $\mu=\mathcal{O}\left(a^{2 k}\right)$ implies the bound (2.6) with any $j>0$.

We will now construct a phase function $\varphi \in C^{1}([0,+\infty))$ such that $\varphi(0)=0$ and $\varphi(r)>0$ for $r>0$. We define the first derivative of $\varphi$ by

$$
\varphi^{\prime}(r)= \begin{cases}\tau(r+1)^{-k}-\tau(a+1)^{-k} & \text { for } 0 \leq r \leq a \\ 0 & \text { for } r \geq a\end{cases}
$$

where

$$
\tau= \begin{cases}\tau_{0} & \text { if } V \text { satisfies (1.3), } \\ \tau_{0} \theta^{2 \alpha / 3} h^{-1 / 3} & \text { if } V \text { satisfies (1.8), }\end{cases}
$$

with some parameter $\tau_{0} \gg 1$ independent of $h$ to be fixed later on. We choose now the parameter $a_{0}$ of the form $a_{0}=\tau_{0}^{\ell}$, where $\ell>0$ is a constant such that $k \ell>2$ and $(\beta-2 k-2 s) \ell>2$. Note that the choice of the parameters $k$ and $s$ guarantees that $\beta-2 k-2 s>0$.

Clearly, the first derivative of $\varphi^{\prime}$ satisfies

$$
\varphi^{\prime \prime}(r)= \begin{cases}-k \tau(r+1)^{-k-1} & \text { for } 0 \leq r<a, \\ 0 & \text { for } r>a .\end{cases}
$$

Lemma 2.2. For all $r \geq 0$ we have the bounds

$$
h^{-1} \varphi(r) \lesssim \begin{cases}h^{-1} & \text { if } V \text { satisfies (1.3), } \\ h^{-4 /(\alpha+3)} \log \left(h^{-1}\right)+1 & \text { if } V \text { satisfies (1.8), }\end{cases}
$$

Proof. The lemma follows from the bounds

$$
\max \varphi=\int_{0}^{a} \varphi^{\prime}(r) d r \leq \tau \int_{0}^{a}(r+1)^{-k} d r \lesssim\left\{\begin{array}{lll}
\tau a^{1-k} & \text { if } \quad k<1 \\
\tau \log a & \text { if } \quad k=1
\end{array}\right.
$$

For $r>0, r \neq a$, set

$$
\begin{gathered}
A(r)=\left(\mu \varphi^{\prime 2}\right)^{\prime}(r), \\
B(r)=B_{1}(r)+B_{2}(r),
\end{gathered}
$$

where

$$
\begin{gathered}
B_{1}(r)=(r+1)^{-\beta} \mu(r)+p(r) \mu^{\prime}(r), \\
B_{2}(r)=\frac{\left(\mu(r) \varphi^{\prime \prime}(r)\right)^{2}}{h^{-1} \varphi^{\prime}(r) \mu(r)+\mu^{\prime}(r)},
\end{gathered}
$$


with $\beta>1$, if $V$ satisfies (1.3), and

$$
\begin{gathered}
B_{1}(r)=\theta^{-1+\alpha}(r+1)^{-\beta} \mu(r)+\left(p(r)+(r+1)^{-\beta}\right) \mu^{\prime}(r), \\
B_{2}(r)=\frac{\left(\mu(r)\left(h^{-1} \theta^{\alpha}(r+1)^{-\beta}+\left|\varphi^{\prime \prime}(r)\right|\right)\right)^{2}}{h^{-1} \varphi^{\prime}(r) \mu(r)+\mu^{\prime}(r)},
\end{gathered}
$$

with $\beta=4$, if $V$ satisfies (1.8). The following lemma will play a crucial role in the proof of the Carleman estimates (4.1) and (4.6) in the case $d \geq 3$.

Lemma 2.3. Given any constant $C>0$ there exists a positive constant $\tau_{1}=\tau_{1}(C, E)$ such that for $\tau$ satisfying (2.7) with $\tau_{0} \geq \tau_{1}$ and for all $0<h \leq 1$ we have the inequality

$$
A(r)-C B(r) \geq-\frac{E}{2} \mu^{\prime}(r)
$$

for all $r>0, r \neq a$.

Proof. For $r<a$ we have

$$
\begin{gathered}
A(r)=-\left((r+1)^{2 k_{0}} \varphi^{\prime 2}\right)^{\prime}+\tau^{2} \partial_{r}\left(1-(r+1)^{k}(a+1)^{-k}\right)^{2} \\
=-2(r+1)^{2 k_{0}} \varphi^{\prime}(r) \varphi^{\prime \prime}(r)-2 k_{0}(r+1)^{2 k_{0}-1} \varphi^{\prime}(r)^{2} \\
\quad-2 k \tau^{2}(r+1)^{k-1}(a+1)^{-k}\left(1-(r+1)^{k}(a+1)^{-k}\right) \\
\geq 2 \tau\left(k-k_{0}\right)(r+1)^{2 k_{0}-k-1} \varphi^{\prime}(r)-2 k \tau^{2}(r+1)^{k-1}(a+1)^{-k} \\
\geq 2 \tau\left(k-k_{0}\right)(r+1)^{2 k_{0}-k-1} \varphi^{\prime}(r)-\mathcal{O}\left(\tau^{2} a^{-k}\right) \mu^{\prime}(r) \\
\geq 2 \tau\left(k-k_{0}\right)(r+1)^{2 k_{0}-k-1} \varphi^{\prime}(r)-\mathcal{O}\left(\tau_{0}^{2} a_{0}^{-k}\right) \mu^{\prime}(r) \\
\geq 2 \tau\left(k-k_{0}\right)(r+1)^{2 k_{0}-k-1} \varphi^{\prime}(r)-\mathcal{O}\left(\tau_{0}^{-k \ell+2}\right) \mu^{\prime}(r) .
\end{gathered}
$$

Hence, taking $\tau_{0}$ large enough, we can arrange the inequality

$$
A(r) \geq 2 \tau\left(k-k_{0}\right)(r+1)^{2 k_{0}-k-1} \varphi^{\prime}(r)-\frac{E}{4} \mu^{\prime}(r)
$$

for all $r<a$. Observe now that if $0<r \leq a / 2$, then

$$
\varphi^{\prime}(r) \geq \gamma \tau(r+1)^{-k}
$$

with some constant $\gamma>0$. By (2.10) and (2.11) we conclude

$$
A(r) \geq \widetilde{\gamma} \tau^{2}(r+1)^{-2\left(k-k_{0}\right)-1}-\frac{E}{4} \mu^{\prime}(r)
$$

for all $r \leq a / 2$ with some constant $\widetilde{\gamma}>0$, and

$$
A(r) \geq-\frac{E}{4} \mu^{\prime}(r) \text { for all } r \neq a .
$$

We will now bound the function $B_{1}$ from above. Since the function $p$ is decreasing, tending to zero, there is $b>0$ such that

$$
p(r)+(r+1)^{-\beta} \leq \frac{E}{9 C} \quad \text { for } \quad r \geq b .
$$

Hence, for every $N>0$ there is a constant $C_{N}>0$ such that we have

$$
\left(p(r)+(r+1)^{-\beta}\right) \mu^{\prime}(r) \leq C_{N}(r+1)^{-N}+\frac{E}{9 C} \mu^{\prime}(r) \text { for all } r \neq a .
$$


Let $0<r<a$. Then $\mu(r)<(r+1)^{2 k}$, and in view of (2.14) with $N$ big enough, we have

$$
B_{1}(r) \leq \widetilde{C}(r+1)^{2 k-\beta}+\frac{E}{9 C} \mu^{\prime}(r),
$$

if $V$ satisfies (1.3), and

$$
B_{1}(r) \leq \widetilde{C} \theta^{-1+\alpha}(r+1)^{2 k-\beta}+\frac{E}{9 C} \mu^{\prime}(r),
$$

with $\beta=4$, if $V$ satisfies (1.8). Observe now that the choice of the parameters $k, k_{0}$ and $\theta$ guarantees that $\beta-2 k \geq 2\left(k-k_{0}\right)+1$ and $\theta^{-1+\alpha}=\theta^{4 \alpha / 3} h^{-2 / 3}$. Therefore, the above inequalities imply

$$
B_{1}(r) \leq \mathcal{O}\left(\tau_{0}^{-2}\right) \tau^{2}(r+1)^{-2\left(k-k_{0}\right)-1}+\frac{E}{9 C} \mu^{\prime}(r) \quad \text { for } \quad r \leq a / 2
$$

in both cases. Similarly, we get

$$
B_{1}(r) \leq \mathcal{O}\left(\tau^{2} a^{-\beta+1}\right) \mu^{\prime}(r)+\frac{E}{9 C} \mu^{\prime}(r) \quad \text { for } \quad a / 2<r<a
$$

and

$$
B_{1}(r) \leq \mathcal{O}\left(\tau^{2} a^{-\beta+2 k+2 s}\right) \mu^{\prime}(r)+\frac{E}{9 C} \mu^{\prime}(r) \quad \text { for } \quad r>a
$$

Since

$$
\tau^{2} a^{-\beta+1}<\tau^{2} a^{-\beta+2 k+2 s} \leq \tau_{0}^{2} a_{0}^{-\beta+2 k+2 s}=\tau_{0}^{-(\beta-2 k-2 s) \ell+2},
$$

we obtain from (2.16) and (2.17),

$$
B_{1}(r) \leq \frac{E}{8 C} \mu^{\prime}(r) \quad \text { for } \quad r>a / 2, r \neq a,
$$

provided $\tau_{0}$ is taken large enough.

We will now bound the function $B_{2}$ from above. We will first consider the case when $V$ satisfies (1.8). Let $0<r \leq a / 2$. In view of (2.11), we have

$$
\begin{aligned}
& B_{2}(r) \lesssim \frac{\mu(r)\left(h^{-2} \theta^{2 \alpha}(r+1)^{-2 \beta}+\varphi^{\prime \prime}(r)^{2}\right)}{h^{-1} \varphi^{\prime}(r)} \\
& \lesssim h^{-1} \theta^{2 \alpha} \frac{\mu(r)(r+1)^{-2 \beta}}{\varphi^{\prime}(r)}+h \frac{\mu(r) \varphi^{\prime \prime}(r)^{2}}{\varphi^{\prime}(r)} \\
& \lesssim \tau^{-1} \theta^{2 \alpha} h^{-1}(r+1)^{3 k-2 \beta}+h \tau(r+1)^{k-2} \\
& \lesssim \tau_{0}^{-3} \tau^{2}(r+1)^{-2\left(k-k_{0}\right)-1}+\tau(r+1)^{k-2}
\end{aligned}
$$

where we have used that $5 k-2 k_{0}<2 \beta-1$. Since $3 k-2 k_{0}-1>0$, we have the inequality

$$
(r+1)^{k-2} \leq b^{3 k-2 k_{0}-1}(r+1)^{-2\left(k-k_{0}\right)-1}+b^{-k-1}(r+1)^{2 k-1}
$$

for every $b>1$. We take $b$ such that $b^{3 k-2 k_{0}-1}=b_{0} \tau$, where $b_{0}>0$ is a small parameter independent of $\tau$ and $h$ to be fixed below. Then the above inequality takes the form

$$
\begin{aligned}
& \tau(r+1)^{k-2} \lesssim b_{0} \tau^{2}(r+1)^{-2\left(k-k_{0}\right)-1}+\tau^{-\frac{2\left(1-k+k_{0}\right)}{3 k-2 k_{0}-1}} \mu^{\prime}(r) \\
& \lesssim b_{0} \tau^{2}(r+1)^{-2\left(k-k_{0}\right)-1}+\tau_{0}^{-1} \mu^{\prime}(r) .
\end{aligned}
$$

Thus, taking $\tau_{0}$ big enough depending on $b_{0}, E$ and $C$, we get the bound

$$
B_{2}(r) \leq \mathcal{O}\left(\tau_{0}^{-1}+b_{0}\right) \tau^{2}(r+1)^{-2\left(k-k_{0}\right)-1}+\frac{E}{8 C} \mu^{\prime}(r) \quad \text { for } \quad 0<r \leq a / 2 .
$$


When $V$ satisfies (1.3) we have $3 k-2 k_{0}-1 \leq 0$, and hence

$$
\tau(r+1)^{k-2} \leq \tau(r+1)^{-2\left(k-k_{0}\right)-1} \leq \tau_{0}^{-1} \tau^{2}(r+1)^{-2\left(k-k_{0}\right)-1} .
$$

Therefore, the inequality (2.19) still holds in this case.

Let us now see that

$$
B_{2}(r) \leq \frac{E}{8 C} \mu^{\prime}(r) \quad \text { for } \quad r>a / 2, r \neq a .
$$

Let $\frac{a}{2}<r<a$. Since in this case $\mu(r) / \mu^{\prime}(r)=\mathcal{O}(r)$, we get the bound

$$
\begin{gathered}
B_{2}(r) \lesssim\left(\frac{\mu(r)}{\mu^{\prime}(r)}\right)^{2}\left(h^{-1} \theta^{\alpha}(r+1)^{-\beta}+\left|\varphi^{\prime \prime}(r)\right|\right)^{2} \mu^{\prime}(r) \\
\lesssim\left(h^{-2} \theta^{2 \alpha}(r+1)^{2-2 \beta}+\tau^{2}(r+1)^{-2 k}\right) \mu^{\prime}(r) \\
\lesssim\left(h^{-2} a^{2-2 \beta}+\tau^{2} a^{-2 k}\right) \mu^{\prime}(r) \\
\lesssim\left(h^{2 m(\beta-1)-2} a_{0}^{2-2 \beta}+h^{2 m-2 / 3} \tau_{0}^{2} a_{0}^{-2 k}\right) \mu^{\prime}(r) \\
\lesssim\left(a_{0}^{2-2 \beta}+\tau_{0}^{2} a_{0}^{-2 k}\right) \mu^{\prime}(r) \lesssim\left(\tau_{0}^{-2 \ell(\beta-1)}+\tau_{0}^{-2 k \ell+2}\right) \mu^{\prime}(r)
\end{gathered}
$$

which clearly implies (2.20) in this case, provided $\tau_{0}$ is taken big enough. Let $r>a$. Using (2.6) with $j=1$, we get

$$
\begin{gathered}
B_{2}(r) \lesssim\left(\frac{\mu(r)}{\mu^{\prime}(r)}\right)^{2}\left(h^{-1} \theta^{\alpha}(r+1)^{-\beta}\right)^{2} \mu^{\prime}(r) \\
\lesssim h^{-2} a^{4 k}(r+1)^{4 s-2 \beta} \mu^{\prime}(r) \\
\lesssim h^{-2} a^{4 k+4 s-2 \beta} \mu^{\prime}(r) \\
\lesssim h^{2 m(\beta-2 k-2 s)-2} a_{0}^{4 k+4 s-2 \beta} \mu^{\prime}(r) \\
\lesssim a_{0}^{4 k+4 s-2 \beta} \mu^{\prime}(r) \lesssim \tau_{0}^{-2 \ell(\beta-2 k-2 s)} \mu^{\prime}(r)
\end{gathered}
$$

which again implies (2.20), provided $\tau_{0}$ is taken big enough. Similarly, in the case when $V$ satisfies (1.3) one concludes that the inequality (2.20) holds for all $r>0, r \neq a$.

It is easy to see that for $r \leq a / 2$ the estimate (2.9) follows from (2.12), (2.15) and (2.19) by taking $b_{0}$ and $\tau_{0}^{-1}$ small enough, while for $r \geq a / 2, r \neq a$, it follows from (2.13), (2.18) and $(2.20)$.

Remark 2. It is easy to see from the proof that when $V$ satisfies (1.8) the inequality (2.9) holds as long as $1 / 2 \leq k \leq 1, k_{0}=k-1 / 2$. The choice $k=1$, however, provides the best resolvent bound in the semiclassical regime, that is, for $0<h \leq h_{0}$ with some constant $0<h_{0} \ll 1$. When $h_{0}<h \leq 1$ the choice of $k$ does not really matter because in this case $g_{s}^{ \pm}(h, \varepsilon)$ is upper bounded by a constant. For example, we may take $k=1 / 2$ and $k_{0}=0$.

The following lemmas will play a crucial role in the proof of the Carleman estimate (4.6) in the case $d=2$.

Lemma 2.4. Given any constants $C, r_{0}>0$ there exists a positive constant $\tau_{1}=\tau_{1}\left(C, E, r_{0}\right)$ such that for $\tau$ satisfying (2.7) with $\tau_{0} \geq \tau_{1}$ and for all $0<h \leq h_{0}, 0<h_{0}<1$ being a constant depending on $E, r_{0}$ and $\tau_{0}$, we have the inequality

$$
A(r)-h^{2} r^{-3} \mu(r)-C B(r) \geq-\frac{2 E}{3} \mu^{\prime}(r)
$$

for all $r \geq r_{0}, r \neq a$. 
Proof. For $r_{0} \leq r<a$ we have

$$
h^{2} r^{-3} \mu(r) \lesssim h^{2}(r+1)^{-3} \mu(r) \lesssim h^{2}(r+1)^{-2} \mu^{\prime}(r) \leq \frac{E}{6} \mu^{\prime}(r),
$$

provided $h$ is taken small enough. For $r>a$, in view of (2.6) with $j=1$, we have

$$
\begin{gathered}
h^{2} r^{-3} \mu(r) \lesssim h^{2} a^{2 k}(r+1)^{2 s-3} \mu^{\prime}(r) \lesssim h^{2} a^{2 k+2 s-3} \mu^{\prime}(r) \\
\lesssim h^{2-m(2 k+2 s-3)} a_{0}^{2 k+2 s-3} \mu^{\prime}(r) \leq \frac{E}{6} \mu^{\prime}(r),
\end{gathered}
$$

provided $h$ is taken small enough, depending on $a_{0}$. Clearly, (2.21) follows from these inequalities and (2.9).

It is easy to see from the proof that when $V$ satisfies (1.3) the inequality (2.21) holds also for $h_{0}<h \leq 1$. This is no longer true when $V$ satisfies (1.8) because in this case $2 k+2 s-3$ does not have the right sign. Therefore, to make (2.21) holds for $h$ not necessarily small, we need to make a new choice of the parameters $k$ and $k_{0}$ in order to change the sign of $2 k+2 s-3$ and for which Lemma 2.3 still holds. Thus, in view of Remark 2 , in the semiclassical regime $\left(0<h \leq h_{0}\right)$ we take $k=1, k_{0}=1 / 2$ and in the classical regime $\left(h_{0}<h \leq 1\right)$ we take $k=1 / 2$, $k_{0}=0$. To cover the second case we need the following

Lemma 2.5. If $V$ satisfies (1.8) we take $k=1 / 2$ and $k_{0}=0$. Then, given any constants $C, r_{0}>0$ there exists a positive constant $\tau_{1}=\tau_{1}\left(C, E, r_{0}\right)$ such that for $\tau$ satisfying (2.7) with $\tau_{0} \geq \tau_{1}$ the inequality (2.21) holds for all $r \geq r_{0}, r \neq a$, and all $0<h \leq 1$.

Proof. For $r_{0} \leq r \leq a / 2$ we have

$$
h^{2} r^{-3} \mu(r) \lesssim(r+1)^{-3} \mu(r) \lesssim(r+1)^{-3+2 k} \lesssim(r+1)^{-2\left(k-k_{0}\right)-1} .
$$

For $a / 2<r<a$ we have

$$
h^{2} r^{-3} \mu(r) \lesssim(r+1)^{-2} \mu^{\prime}(r) \lesssim a^{-2} \mu^{\prime}(r) \lesssim a_{0}^{-2} \mu^{\prime}(r) \leq \frac{E}{6} \mu^{\prime}(r),
$$

provided $a_{0}$ is taken big enough. For $r>a$ we have

$$
h^{2} r^{-3} \mu(r) \lesssim a^{2 k}(r+1)^{2 s-3} \mu^{\prime}(r) \lesssim a^{2 k+2 s-3} \mu^{\prime}(r) \lesssim a_{0}^{2 k+2 s-3} \mu^{\prime}(r) \leq \frac{E}{6} \mu^{\prime}(r),
$$

provided $a_{0}$ is taken big enough. Then it is easy to see that (2.21) follows from these inequalities and Remark 2.

\section{Carleman estimates for Hölder potentials on Bounded domains}

Throughout this section $X \subset \mathbb{R}^{d}, d \geq 2$, will be a bounded, connected domain with a smooth boundary $\partial X$. Introduce the operator

$$
P(h)=-h^{2} \Delta+V(x)
$$

where $0<h \leq 1$ is a semiclassical parameter and $V \in L^{\infty}(X)$ is a real-valued potential. Let $U \subset X, U \neq \emptyset$, be an arbitrary open domain, independent of $h$, such that $\partial U \cap \partial X=\emptyset$ and let $z \in \mathbb{C},|z| \leq C_{0}, C_{0}>0$ being a constant independent of $h$. We will also denote by $H_{h}^{1}$ the Sobolev space equipped with the semiclassical norm. Given any $0<\alpha \leq 1$, denote by $C^{\alpha}(\bar{X})$ the space of all functions $a$ such that

$$
\|a\|_{C^{\alpha}}:=\sup _{x^{\prime}, x \in \bar{X}: 0<\left|x-x^{\prime}\right| \leq 1} \frac{\left|a(x)-a\left(x^{\prime}\right)\right|}{\left|x-x^{\prime}\right|^{\alpha}}<+\infty .
$$

We have the following 
Theorem 3.1. Let $V \in C^{\alpha}(\bar{X})$ with $0<\alpha \leq 1$. Then, there exists a positive constant $\gamma$ depending on $U,\|V\|_{C^{\alpha}}$ and $C_{0}$ but independent of $h$ such that for all $0<h \leq 1$ we have the estimate

$$
\|u\|_{H_{h}^{1}(X)} \leq e^{\gamma h^{-4 /(\alpha+3)}}\|(P(h)-z) u\|_{L^{2}(X)}+e^{\gamma h^{-4 /(\alpha+3)}}\|u\|_{H_{h}^{1}(U)}
$$

for every $u \in H^{2}(X)$ such that $\left.u\right|_{\partial X}=0$.

It is proved in Section 2 of [14] that for complex-valued potentials $V \in L^{\infty}(X)$ the estimate (3.1) holds with $\alpha=0$. The proof is based on the local Carleman estimates proved in [8]. We will follow the same strategy in the case of Hölder potentials as well. For such potentials we will get new local Carleman estimates by making use of the results of [8]. To be more precise, we let $W \subset X$ be a small open domain and let $x$ be local coordinates in $W$. If $\Gamma:=\bar{W} \cap \partial X$ is not empty we choose $x=\left(x_{1}, x^{\prime}\right), x_{1}>0$ being the normal coordinate in $W$ and $x^{\prime}$ the tangential ones. Thus in these coordinates $\Gamma$ is given by $\left\{x_{1}=0\right\}$. Let $\phi, \phi_{1} \in C^{\infty}(\bar{W})$ be real-valued functions such that $\operatorname{supp} \phi \subset \operatorname{supp} \phi_{1} \subset \bar{W}, \phi_{1}=1$ on supp $\phi$. When $V \in C^{\alpha}(\bar{X})$ with $0<\alpha<1$ we approximate the function $\phi_{1} V$ by the smooth function

$$
V_{\theta}(x)=\theta^{-1} \int_{X} \varrho\left(\left(x^{\prime}-x\right) / \theta\right)\left(\phi_{1} V\right)\left(x^{\prime}\right) d x^{\prime}
$$

where $\varrho \in C_{0}^{\infty}(|x| \leq 1)$ is a real-valued function such that $\int_{\mathbb{R}^{d}} \varrho(x) d x=1$ and $0<\theta<1$ is a small parameter to be fixed later on. The fact that $V \in C^{\alpha}(\bar{X})$ implies the bounds

$$
\begin{gathered}
\left|\left(\phi_{1} V\right)(x)-V_{\theta}(x)\right| \lesssim \theta^{\alpha}, \\
\left|\partial_{x}^{\beta} V_{\theta}(x)\right| \lesssim \theta^{\alpha-1},
\end{gathered}
$$

for all multi-indices $\beta$ such that $|\beta|=1$. Set $\widetilde{V}=\theta^{1-\alpha}\left(V_{\theta}-z\right)$ if $V \in C^{\alpha}(\bar{X})$ with $0<\alpha<1$, $\widetilde{V}=V-z$ if $V \in C^{1}(\bar{X})$. In view of (3.2) and (3.3) we have $\partial_{x}^{\beta} \widetilde{V}(x)=\mathcal{O}(1)$ uniformly in $\theta$, for all multi-indices $\beta$ such that $|\beta| \leq 1$.

Let now $\psi \in C^{\infty}(\bar{W})$ be a real-valued function independent of $h$ and $\theta$ such that

$$
\nabla \psi \neq 0 \quad \text { in } \quad \bar{W} .
$$

If $\Gamma \neq \emptyset$ we also suppose that

$$
\frac{\partial \psi}{\partial x_{1}}\left(0, x^{\prime}\right)>0 \text { for all } x^{\prime}
$$

We set $\varphi=e^{\lambda \psi}$, where $\lambda>0$ is a big parameter to be fixed later on, independent of $h$ and $\theta$. Let $p(x, \xi) \in C^{\infty}\left(T^{*} W\right)$ be the principal symbol of the operator $-\Delta$ and let $0<\widetilde{h} \ll 1$ be a new semiclassical parameter. Then the principal symbol, $\widetilde{p}_{\varphi}$, of the operator

$$
e^{\varphi / \widetilde{h}}\left(-\widetilde{h}^{2} \Delta+\widetilde{V}\right) e^{-\varphi / \widetilde{h}}
$$

is given by the formula

$$
\widetilde{p}_{\varphi}(x, \xi)=p(x, \xi+i \nabla \varphi(x))+\widetilde{V}(x) .
$$

An easy computation shows that given any constant $C>0$ there is $\lambda=\lambda(C)$ such that the condition (3.4) for the function $\psi$ implies the following condition for the function $\varphi$ :

$$
\left\{\operatorname{Re} \widetilde{p}_{\varphi}, \operatorname{Im} \tilde{p}_{\varphi}\right\}(x, \xi) \geq c_{1} \quad \text { for } \quad|\xi| \leq C,
$$

with some constant $c_{1}>0$ independent of $\theta$. On the other hand, if $C$ is taken large enough we can arrange the lower bound

$$
\left|\widetilde{p}_{\varphi}(x, \xi)\right| \geq c_{2}|\xi|^{2} \quad \text { for } \quad|\xi| \geq C,
$$


with some constant $c_{2}>0$ independent of $\theta$. If $\Gamma \neq \emptyset$ the condition (3.5) implies

$$
\frac{\partial \varphi}{\partial x_{1}}\left(0, x^{\prime}\right)>0 \text { for all } x^{\prime}
$$

Now we are in position to use Propositions 1 and 2 of [8], where the proof is based on the properties (3.6), (3.7) and (3.8). We have the following

Proposition 3.2. Let the function $u$ be as in Theorem 3.1. Then there exist constants $C_{1}, \widetilde{h}_{0}>0$ such that for all $0<\widetilde{h} \leq \widetilde{h}_{0}$ we have the estimate

$$
\int_{X}\left(|\phi u|^{2}+|\widetilde{h} \nabla(\phi u)|^{2}\right) e^{2 \varphi / \widetilde{h}} d x \leq C_{1} \widetilde{h}^{-1} \int_{X}\left|\left(-\widetilde{h}^{2} \Delta+\widetilde{V}\right)(\phi u)\right|^{2} e^{2 \varphi / \widetilde{h}} d x .
$$

We take $\widetilde{h}=h \theta^{(1-\alpha) / 2}$ when $\alpha<1$ and we rewrite the inequality (3.9) as follows

$$
\begin{gathered}
\int_{X}\left(|\phi u|^{2}+\theta^{1-\alpha}|h \nabla(\phi u)|^{2}\right) e^{2 \varphi / h \theta^{(1-\alpha) / 2}} d x \\
\leq C_{1} h^{-1} \theta^{3(1-\alpha) / 2} \int_{X}\left|\left(-h^{2} \Delta+V_{\theta}-z\right)(\phi u)\right|^{2} e^{2 \varphi / h \theta^{(1-\alpha) / 2}} d x \\
\leq C_{1} h^{-1} \theta^{3(1-\alpha) / 2} \int_{X}|(P(h)-z)(\phi u)|^{2} e^{2 \varphi / h \theta^{(1-\alpha) / 2}} d x \\
+C_{1} h^{-1} \theta^{3(1-\alpha) / 2} \sup \left|\phi_{1} V-V_{\theta}\right|^{2} \int_{X}|\phi u|^{2} e^{2 \varphi / h \theta^{(1-\alpha) / 2}} d x \\
\leq C_{1} h^{-1} \theta^{3(1-\alpha) / 2} \int_{X}|(P(h)-z)(\phi u)|^{2} e^{2 \varphi / h \theta^{(1-\alpha) / 2}} d x \\
+C_{2} h^{-1} \theta^{(3+\alpha) / 2} \int_{X}|\phi u|^{2} e^{2 \varphi / h \theta^{(1-\alpha) / 2}} d x .
\end{gathered}
$$

We now take $\theta=h^{2 /(\alpha+3)} \kappa^{2 /(1-\alpha)}$, where $\kappa>0$ is a small parameter independent of $h$. Thus, taking $\kappa$ small enough we can absorb the last term in the right-hand side of the above inequality. When $\alpha=1$ we take $\widetilde{h}=h \kappa$. Thus we deduce from Proposition 3.2 the following

Proposition 3.3. Let the function $u$ be as in Theorem 3.1. Then there exist constants $\widetilde{C}, \kappa_{0}>0$ such that for all $0<\kappa \leq \kappa_{0}$ and all $0<h \leq 1$ we have the estimate

$$
\begin{gathered}
\int_{X}\left(|\phi u|^{2}+|h \nabla(\phi u)|^{2}\right) e^{2 \varphi / \kappa h^{4 /(\alpha+3)}} d x \\
\leq \widetilde{C} \kappa h^{-2(\alpha+1) /(\alpha+3)} \int_{X}|(P(h)-z)(\phi u)|^{2} e^{2 \varphi / \kappa h^{4 /(\alpha+3)}} d x .
\end{gathered}
$$

Now Theorem 3.1 follows from Proposition 3.3 in precisely the same way as in Section 2 of [14], where the analysis is carried out in the particular case $\alpha=0$. It is an easy observation that the general case requires no changes in the arguments, and therefore we omit the details. 


\section{Resolvent estimates}

The following global Carlemann estimate is similar to that one in Section 3 of [13] and can be proved in the same way. The proof will be carried out in Section 5. In what follows we set $\mathcal{D}_{r}=-i h \partial_{r}$.

Theorem 4.1. Let $d \geq 3$ and let the potential $V$ satisfy (1.1). Let also $V$ satisfy either (1.3) or (1.8) and let $s$ satisfy (2.4). Then, for all $0<h \leq 1,0<\varepsilon \leq 1$ and for all functions $f \in H^{2}\left(\mathbb{R}^{d}\right)$ such that

$$
(|x|+1)^{s}(P(h)-E \pm i \varepsilon) f \in L^{2}\left(\mathbb{R}^{d}\right)
$$

we have the estimate

$$
\begin{gathered}
\left\|(|x|+1)^{-s} e^{\varphi / h} f\right\|_{L^{2}\left(\mathbb{R}^{d}\right)}+\left\|(|x|+1)^{-s} e^{\varphi / h} \mathcal{D}_{r} f\right\|_{L^{2}\left(\mathbb{R}^{d}\right)} \\
\leq C a^{2} h^{-1}\left\|(|x|+1)^{s} e^{\varphi / h}(P(h)-E \pm i \varepsilon) f\right\|_{L^{2}\left(\mathbb{R}^{d}\right)} \\
+C \tau a(\varepsilon / h)^{1 / 2}\left\|e^{\varphi / h} f\right\|_{L^{2}\left(\mathbb{R}^{d}\right)}
\end{gathered}
$$

with a constant $C>0$ independent of $h, \varepsilon$ and $f$.

Theorems 1.1 and 1.2 can be obtained from Theorem 4.1 in the same way as in Section 4 of [13]. We will sketch the proof for the sake of completeness. It follows from the estimate (4.1) and Lemma 2.2 that for $0<h \leq 1$ and $s$ satisfying (2.4) we have the estimate

$$
\left\|(|x|+1)^{-s} f\right\|_{L^{2}} \leq M\left\|(|x|+1)^{s}(P(h)-E \pm i \varepsilon) f\right\|_{L^{2}}+M \varepsilon^{1 / 2}\|f\|_{L^{2}}
$$

where $M>0$ is given by

$$
\log M= \begin{cases}C h^{-1} & \text { if } V \text { satisfies (1.3), } \\ C h^{-4 /(\alpha+3)} \log \left(h^{-1}\right)+C & \text { if } V \text { satisfies (1.8) }\end{cases}
$$

with a constant $C>0$ independent of $h$ and $\varepsilon$. On the other hand, since the operator $P(h)$ is symmetric, we have

$$
\begin{gathered}
\varepsilon\|f\|_{L^{2}}^{2}= \pm \operatorname{Im}\langle(P(h)-E \pm i \varepsilon) f, f\rangle_{L^{2}} \\
\leq(2 M)^{-2}\left\|(|x|+1)^{-s} f\right\|_{L^{2}}^{2}+(2 M)^{2}\left\|(|x|+1)^{s}(P(h)-E \pm i \varepsilon) f\right\|_{L^{2}}^{2}
\end{gathered}
$$

which yields

$$
M \varepsilon^{1 / 2}\|f\|_{L^{2}} \leq \frac{1}{2}\left\|(|x|+1)^{-s} f\right\|_{L^{2}}+2 M^{2}\left\|(|x|+1)^{s}(P(h)-E \pm i \varepsilon) f\right\|_{L^{2}} .
$$

By (4.2) and (4.3) we get

$$
\left\|(|x|+1)^{-s} f\right\|_{L^{2}} \leq 4 M^{2}\left\|(|x|+1)^{s}(P(h)-E \pm i \varepsilon) f\right\|_{L^{2}} .
$$

It follows from (4.4) that the resolvent estimate

$$
\left\|(|x|+1)^{-s}(P(h)-E \pm i \varepsilon)^{-1}(|x|+1)^{-s}\right\|_{L^{2} \rightarrow L^{2}} \leq 4 M^{2}
$$

holds for all $0<h \leq 1$ and $s$ satisfying (2.4), and hence for all $s>1 / 2$ independent of $h$. Clearly, (4.5) implies the desired bounds for $g_{s}^{ \pm}$.

Given any $r_{0}>0$ we denote $Y_{r_{0}}:=\left\{x \in \mathbb{R}^{d}:|x| \geq r_{0}\right\}$ and we let $\eta_{r_{0}} \in C^{\infty}(\mathbb{R})$ be such that $\eta_{r_{0}}(r)=0$ for $r \leq r_{0} / 3, \eta_{r_{0}}(r)=1$ for $r \geq r_{0} / 2$. We set $V_{\eta}(x):=\eta_{r_{0}}(|x|) V(x)$. To prove Theorem 1.3 we need the following 
Theorem 4.2. Let $d \geq 3$ and let the potential $V$ satisfy (1.1) for $|x| \geq r_{0}$. Let also $V_{\eta}$ satisfy either (1.3) or (1.8) and let s satisfy (2.4). Then, for all $0<h \leq 1,0<\varepsilon \leq 1$ and for all functions $f \in H^{2}\left(Y_{r_{0}}\right)$ such that $f=\partial_{r} f=0$ on $\partial Y_{r_{0}}$ and

$$
(|x|+1)^{s}(P(h)-E \pm i \varepsilon) f \in L^{2}\left(Y_{r_{0}}\right)
$$

we have the estimate

$$
\begin{gathered}
\left\|(|x|+1)^{-s} e^{\varphi / h} f\right\|_{L^{2}\left(Y_{r_{0}}\right)}+\left\|(|x|+1)^{-s} e^{\varphi / h} \mathcal{D}_{r} f\right\|_{L^{2}\left(Y_{r_{0}}\right)} \\
\leq C a^{2} h^{-1}\left\|(|x|+1)^{s} e^{\varphi / h}(P(h)-E \pm i \varepsilon) f\right\|_{L^{2}\left(Y_{r_{0}}\right)} \\
+C \tau a(\varepsilon / h)^{1 / 2}\left\|e^{\varphi / h} f\right\|_{L^{2}\left(Y_{r_{0}}\right)}
\end{gathered}
$$

with a constant $C>0$ independent of $h, \varepsilon$ and $f$.

Let $d=2$. If $V_{\eta}$ satisfies (1.8) and $k=1, k_{0}=1 / 2$, then (4.6) holds for $0<h \leq h_{0}$ with some constant $0<h_{0} \ll 1$ depending on $\tau_{0}$. If $V_{\eta}$ satisfies (1.8) and $k=1 / 2, k_{0}=0$, or $V_{\eta}$ satisfies (1.3), then (4.6) holds for all $0<h \leq 1$.

The proof of Theorem 4.2 is similar to that one of Theorem 4.1 with some suitable modifications when $d=2$ and will be carried out in Section 5 .

Theorem 1.3 can be derived from Theorems 3.1 and 4.2 in a way similar to the one developed in Section 5 of [14]. Let $r_{0}>0$ be such that $Y_{r_{0} / 3} \subset \Omega$. Fix $r_{j}, j=1,2,3,4$, such that $r_{0}<r_{1}<r_{2}<r_{3}<r_{4}$. Choose functions $\psi_{1}, \psi_{2} \in C^{\infty}\left(\mathbb{R}^{d}\right)$, depending only on the radial variable $r$, such that $\psi_{1}=1$ in $\mathbb{R}^{d} \backslash Y_{r_{1}}, \psi_{1}=0$ in $Y_{r_{2}}, \psi_{2}=1$ in $\mathbb{R}^{d} \backslash Y_{r_{3}}, \psi_{2}=0$ in $Y_{r_{4}}$. If $s$ satisfies (2.4), we choose a function $\chi_{s} \in C^{\infty}(\bar{\Omega}), \chi_{s}>0$, such that $\chi_{s}(x)=|x|^{-s}$ on $Y_{r_{0}}$. Let $f \in H^{2}(\Omega)$ be such that $\chi_{s}^{-1}(P(h)-E \pm i \varepsilon) f \in L^{2}(\Omega)$ and $\left.f\right|_{\partial \Omega}=0$. Set

and observe that

$$
\begin{gathered}
\mathcal{Q}_{0}=\left\|\chi_{s}^{-1}(P(h)-E \pm i \varepsilon) f\right\|_{L^{2}(\Omega)}, \\
\mathcal{Q}_{1}=\|f\|_{L^{2}\left(Y_{r_{1}} \backslash Y_{r_{2}}\right)}+\left\|\mathcal{D}_{r} f\right\|_{L^{2}\left(Y_{r_{1}} \backslash Y_{r_{2}}\right)}, \\
\mathcal{Q}_{2}=\|f\|_{L^{2}\left(Y_{r_{3}} \backslash Y_{r_{4}}\right)}+\left\|\mathcal{D}_{r} f\right\|_{L^{2}\left(Y_{r_{3}} \backslash Y_{r_{4}}\right)},
\end{gathered}
$$

$$
\left\|\left[P(h), \psi_{j}\right] f\right\|_{L^{2}} \lesssim \mathcal{Q}_{j}, \quad j=1,2 .
$$

We now apply Theorem 3.1 to the function $\psi_{2} f$ with $X=\Omega \backslash Y_{r_{4}}$ and $U \subset X$ such that $U \cap \operatorname{supp} \psi_{2}=\emptyset$. Thus we obtain

$$
\begin{gathered}
\|f\|_{H_{h}^{1}\left(\Omega \backslash Y_{r_{3}}\right)} \leq\left\|\psi_{2} f\right\|_{H_{h}^{1}\left(\Omega \backslash Y_{r_{4}}\right)} \\
\leq e^{\gamma h^{-4 /(\alpha+3)}}\left\|(P(h)-E \pm i \varepsilon) \psi_{2} f\right\|_{L^{2}\left(\Omega \backslash Y_{r_{4}}\right)} \\
\leq e^{\gamma h^{-4 /(\alpha+3)}}\|(P(h)-E \pm i \varepsilon) f\|_{L^{2}\left(\Omega \backslash Y_{r_{4}}\right)}+e^{\gamma h^{-4 /(\alpha+3)}} \mathcal{Q}_{2}
\end{gathered}
$$

with a constant $\gamma>0$ independent of $h$ and $\tau_{0}$. In particular, (4.7) implies

$$
\mathcal{Q}_{1} \leq e^{\gamma h^{-4 /(\alpha+3)}} \mathcal{Q}_{0}+e^{\gamma h^{-4 /(\alpha+3)}} \mathcal{Q}_{2} .
$$

On the other hand, it is clear that if $V$ satisfies (1.10) with $\alpha=1$ and $\beta>1$ (resp. $0<\alpha<1$ and $\beta=4$ ), then $V_{\eta}$ satisfies (1.3) (resp. (1.8)). Therefore, we can apply Theorem 4.2 to the function $\left(1-\psi_{1}\right) f$ to obtain

$$
\begin{gathered}
\left\|(|x|+1)^{-s} e^{\varphi / h} f\right\|_{L^{2}\left(Y_{r_{2}}\right)}+\left\|(|x|+1)^{-s} e^{\varphi / h} \mathcal{D}_{r} f\right\|_{L^{2}\left(Y_{r_{2}}\right)} \\
\leq\left\|(|x|+1)^{-s} e^{\varphi / h}\left(1-\psi_{1}\right) f\right\|_{L^{2}\left(Y_{r_{1}}\right)}+\left\|(|x|+1)^{-s} e^{\varphi / h} \mathcal{D}_{r}\left(1-\psi_{1}\right) f\right\|_{L^{2}\left(Y_{r_{1}}\right)} \\
\leq C a^{2} h^{-1}\left\|(|x|+1)^{s} e^{\varphi / h}(P(h)-E \pm i \varepsilon)\left(1-\psi_{1}\right) f\right\|_{L^{2}\left(Y_{r_{1}}\right)}
\end{gathered}
$$




$$
\begin{gathered}
+C \tau a(\varepsilon / h)^{1 / 2}\left\|e^{\varphi / h} f\right\|_{L^{2}\left(Y_{r_{1}}\right)} \\
\leq C a^{2} h^{-1}\left\|(|x|+1)^{s} e^{\varphi / h}(P(h)-E \pm i \varepsilon) f\right\|_{L^{2}\left(Y_{r_{1}}\right)}+C a^{2} h^{-1} e^{\varphi\left(r_{2}\right) / h} \mathcal{Q}_{1} \\
+C \tau a(\varepsilon / h)^{1 / 2}\left\|e^{\varphi / h} f\right\|_{L^{2}\left(Y_{r_{1}}\right)}
\end{gathered}
$$

for all $0<h \leq 1$. In particular, (4.9) implies

$$
\begin{gathered}
e^{\varphi\left(r_{3}\right) / h} \mathcal{Q}_{2} \leq C a^{2} h^{-1} e^{\max \varphi / h} \mathcal{Q}_{0}+C \tau a(\varepsilon / h)^{1 / 2} e^{\max \varphi / h}\|f\|_{L^{2}(\Omega)} \\
+C a^{2} h^{-1} e^{\varphi\left(r_{2}\right) / h} \mathcal{Q}_{1} .
\end{gathered}
$$

We have

$$
\varphi\left(r_{3}\right)-\varphi\left(r_{2}\right)=\tau \int_{r_{2}}^{r_{3}}\left((r+1)^{-k}-(a+1)^{-k}\right) d r \geq c \tau
$$

with some constant $c>0$. We deduce from (4.10)

$$
\begin{gathered}
\mathcal{Q}_{2} \leq \exp \left(\widetilde{\beta} h^{-4 /(\alpha+3)}+\max \varphi / h\right) \mathcal{Q}_{0} \\
+\varepsilon^{1 / 2} \exp \left(\widetilde{\beta} h^{-4 /(\alpha+3)}+\max \varphi / h\right)\|f\|_{L^{2}(\Omega)} \\
+\tau_{0}^{2 \ell} \exp \left(\left(\beta-c \tau_{0}\right) h^{-4 /(\alpha+3)}\right) \mathcal{Q}_{1}
\end{gathered}
$$

with a constant $\widetilde{\beta}>0$ independent of $h$ and a constant $\beta>0$ independent of $h$ and $\tau_{0}$. Combining (4.8) and (4.11) we get

$$
\begin{gathered}
\mathcal{Q}_{2} \leq \exp \left((\widetilde{\beta}+\gamma) h^{-4 /(\alpha+3)}+\max \varphi / h\right) \mathcal{Q}_{0} \\
+\varepsilon^{1 / 2} \exp \left(\widetilde{\beta} h^{-4 /(\alpha+3)}+\max \varphi / h\right)\|f\|_{L^{2}(\Omega)} \\
+\tau_{0}^{2 \ell} \exp \left(\left(\beta+\gamma-c \tau_{0}\right) h^{-4 /(\alpha+3)}\right) \mathcal{Q}_{2} .
\end{gathered}
$$

Taking $\tau_{0}$ big enough, independent of $h$, we can arrange that

$$
\tau_{0}^{2 \ell} \exp \left(\left(\beta+\gamma-c \tau_{0}\right) h^{-4 /(\alpha+3)}\right) \leq \tau_{0}^{2 \ell} \exp \left(-c \tau_{0} h^{-4 /(\alpha+3)} / 2\right) \leq \tau_{0}^{2 \ell} \exp \left(-c \tau_{0} / 2\right) \leq 1 / 2
$$

for all $0<h \leq 1$. Thus we can absorb the last term in the right-hand side of (4.12) to conclude that

$$
\begin{aligned}
& \mathcal{Q}_{1}+\mathcal{Q}_{2} \leq \exp \left(\beta_{1} h^{-4 /(\alpha+3)}+\max \varphi / h\right) \mathcal{Q}_{0} \\
& +\varepsilon^{1 / 2} \exp \left(\beta_{1} h^{-4 /(\alpha+3)}+\max \varphi / h\right)\|f\|_{L^{2}(\Omega)}
\end{aligned}
$$

with a constant $\beta_{1}>0$ independent of $h$. By (4.7), (4.9) and (4.13) we obtain

$$
\left\|\chi_{s} f\right\|_{L^{2}(\Omega)} \leq N \mathcal{Q}_{0}+\varepsilon^{1 / 2} N\|f\|_{L^{2}(\Omega)}
$$

where

$$
N=\exp \left(\beta_{2} h^{-4 /(\alpha+3)}+\max \varphi / h\right)
$$

with a constant $\beta_{2}>0$ independent of $h$. In the same way as above, using the fact that the operator $P(h)$ is symmetric, we get from (4.14) that the resolvent estimate

$$
\left\|\chi_{s}(P(h)-E \pm i \varepsilon)^{-1} \chi_{s}\right\|_{L^{2}(\Omega) \rightarrow L^{2}(\Omega)} \leq 4 N^{2}
$$

holds for all $0<h \leq 1,0<\varepsilon \leq 1$ and $s$ satisfying (2.4), which together with Lemma 2.2 clearly imply the desired bound. 


\section{Proof of Theorems 4.1 And 4.2}

The main point is to work with the polar coordinates $(r, w) \in \mathbb{R}^{+} \times \mathbb{S}^{d-1}, r=|x|, w=x /|x|$ and to use that $L^{2}\left(\mathbb{R}^{d}\right)=L^{2}\left(\mathbb{R}^{+} \times \mathbb{S}^{d-1}, r^{d-1} d r d w\right)$. In what follows in this section we denote by $\|\cdot\|$ and $\langle\cdot, \cdot\rangle$ the norm and the scalar product in $L^{2}\left(\mathbb{S}^{d-1}\right)$. We will make use of the identity

$$
r^{(d-1) / 2} \Delta r^{-(d-1) / 2}=\partial_{r}^{2}+\frac{\widetilde{\Delta}_{w}}{r^{2}}
$$

where $\widetilde{\Delta}_{w}=\Delta_{w}-\frac{1}{4}(d-1)(d-3)$ and $\Delta_{w}$ denotes the negative Laplace-Beltrami operator on $\mathbb{S}^{d-1}$. Set $u=r^{(d-1) / 2} e^{\varphi / h} f$ and

$$
\begin{gathered}
\mathcal{P}^{ \pm}(h)=r^{(d-1) / 2}(P(h)-E \pm i \varepsilon) r^{-(d-1) / 2}, \\
\mathcal{P}_{\varphi}^{ \pm}(h)=e^{\varphi / h} \mathcal{P}^{ \pm}(h) e^{-\varphi / h} .
\end{gathered}
$$

Using (5.1) we can write the operator $\mathcal{P}^{ \pm}(h)$ in the coordinates $(r, w)$ as follows

$$
\mathcal{P}^{ \pm}(h)=\mathcal{D}_{r}^{2}+\frac{\Lambda_{w}}{r^{2}}-E \pm i \varepsilon+V
$$

where we have put $\mathcal{D}_{r}=-i h \partial_{r}$ and $\Lambda_{w}=-h^{2} \widetilde{\Delta}_{w}$. Since the function $\varphi$ depends only on the variable $r$, we get

$$
\mathcal{P}_{\varphi}^{ \pm}(h)=\mathcal{D}_{r}^{2}+\frac{\Lambda_{w}}{r^{2}}-E \pm i \varepsilon-\varphi^{\prime 2}+h \varphi^{\prime \prime}+2 i \varphi^{\prime} \mathcal{D}_{r}+V .
$$

We write $V=V_{L}+V_{S}$ with $V_{L}:=V_{\theta}$ and $V_{S}:=V-V_{\theta}$ if $V$ satisfies (1.8), and $V_{L}:=V$ and $V_{S}:=0$ if $V$ satisfies (1.3). For $r>0, r \neq a$, introduce the function

$$
F(r)=-\left\langle\left(r^{-2} \Lambda_{w}-E-\varphi^{\prime}(r)^{2}+V_{L}(r, \cdot)\right) u(r, \cdot), u(r, \cdot)\right\rangle+\left\|\mathcal{D}_{r} u(r, \cdot)\right\|^{2}
$$

where $V_{L}(r, w):=V_{L}(r w)$. Then its first derivative is given by

$$
\begin{gathered}
F^{\prime}(r)=\frac{2}{r}\left\langle r^{-2} \Lambda_{w} u(r, \cdot), u(r, \cdot)\right\rangle+\left(\left(\varphi^{\prime}\right)^{2}-V_{L}\right)^{\prime}\|u(r, \cdot)\|^{2} \\
\quad-2 h^{-1} \operatorname{Im}\left\langle\mathcal{P}_{\varphi}^{ \pm}(h) u(r, \cdot), \mathcal{D}_{r} u(r, \cdot)\right\rangle \\
\pm 2 \varepsilon h^{-1} \operatorname{Re}\left\langle u(r, \cdot), \mathcal{D}_{r} u(r, \cdot)\right\rangle+4 h^{-1} \varphi^{\prime}\left\|\mathcal{D}_{r} u(r, \cdot)\right\|^{2} \\
+2 h^{-1} \operatorname{Im}\left\langle\left(V_{S}+h \varphi^{\prime \prime}\right) u(r, \cdot), \mathcal{D}_{r} u(r, \cdot)\right\rangle .
\end{gathered}
$$

Thus we obtain the identity

$$
\begin{gathered}
(\mu F)^{\prime}=\mu^{\prime} F+\mu F^{\prime} \\
=\left(2 r^{-1} \mu-\mu^{\prime}\right)\left\langle r^{-2} \Lambda_{w} u(r, \cdot), u(r, \cdot)\right\rangle \\
+\left(E \mu^{\prime}+\left(\mu\left(\varphi^{\prime}\right)^{2}-\mu V_{L}\right)^{\prime}\right)\|u(r, \cdot)\|^{2} \\
-2 h^{-1} \mu \operatorname{Im}\left\langle\mathcal{P}_{\varphi}^{ \pm}(h) u(r, \cdot), \mathcal{D}_{r} u(r, \cdot)\right\rangle \\
\pm 2 \varepsilon h^{-1} \mu \operatorname{Re}\left\langle u(r, \cdot), \mathcal{D}_{r} u(r, \cdot)\right\rangle+\left(\mu^{\prime}+4 h^{-1} \varphi^{\prime} \mu\right)\left\|\mathcal{D}_{r} u(r, \cdot)\right\|^{2} \\
+2 h^{-1} \mu \operatorname{Im}\left\langle\left(V_{S}+h \varphi^{\prime \prime}\right) u(r, \cdot), \mathcal{D}_{r} u(r, \cdot)\right\rangle .
\end{gathered}
$$

Using that $\Lambda_{w} \geq 0$ as long as $d \geq 3$ together with (2.5) we get the inequality

$$
\begin{gathered}
\mu^{\prime} F+\mu F^{\prime} \geq\left(E \mu^{\prime}+\left(\mu\left(\varphi^{\prime}\right)^{2}-\mu V_{L}\right)^{\prime}\right)\|u(r, \cdot)\|^{2} \\
+\left(\mu^{\prime}+4 h^{-1} \varphi^{\prime} \mu\right)\left\|\mathcal{D}_{r} u(r, \cdot)\right\|^{2} \\
-\frac{3 h^{-2} \mu^{2}}{\mu^{\prime}}\left\|\mathcal{P}_{\varphi}^{ \pm}(h) u(r, \cdot)\right\|^{2}-\frac{\mu^{\prime}}{3}\left\|\mathcal{D}_{r} u(r, \cdot)\right\|^{2} \\
-\varepsilon h^{-1} \mu\left(\|u(r, \cdot)\|^{2}+\left\|\mathcal{D}_{r} u(r, \cdot)\right\|^{2}\right)
\end{gathered}
$$


where

$$
\begin{gathered}
-3 h^{-2} \mu^{2}\left(\mu^{\prime}+4 h^{-1} \varphi^{\prime} \mu\right)^{-1}\left\|\left(V_{S}+h \varphi^{\prime \prime}\right) u(r, \cdot)\right\|^{2} \\
-\frac{1}{3}\left(\mu^{\prime}+4 h^{-1} \varphi^{\prime} \mu\right)\left\|\mathcal{D}_{r} u(r, \cdot)\right\|^{2} \\
\geq\left(E \mu^{\prime}+\left(\mu\left(\varphi^{\prime}\right)^{2}\right)^{\prime}-T_{L} \mu-Z_{L} \mu^{\prime}\right)\|u(r, \cdot)\|^{2} \\
+\frac{\mu^{\prime}}{3}\left\|\mathcal{D}_{r} u(r, \cdot)\right\|^{2}-\frac{3 h^{-2} \mu^{2}}{\mu^{\prime}}\left\|\mathcal{P}_{\varphi}^{ \pm}(h) u(r, \cdot)\right\|^{2} \\
-\varepsilon h^{-1} \mu\left(\|u(r, \cdot)\|^{2}+\left\|\mathcal{D}_{r} u(r, \cdot)\right\|^{2}\right) \\
-3 h^{-2} \mu^{2}\left(\mu^{\prime}+4 h^{-1} \varphi^{\prime} \mu\right)^{-1}\left(Q_{S}+h\left|\varphi^{\prime \prime}\right|\right)^{2}\|u(r, \cdot)\|^{2}
\end{gathered}
$$

if $V$ satisfies (1.3),

$$
T_{L}=\mathcal{O}\left((r+1)^{-\beta}\right), \quad Z_{L}=p(r), \quad Q_{S}=0,
$$

$$
T_{L}=\mathcal{O}\left(\theta^{-1+\alpha}(r+1)^{-4}\right), \quad Z_{L}=p(r)+\mathcal{O}\left((r+1)^{-4}\right), \quad Q_{S}=\mathcal{O}\left(\theta^{\alpha}(r+1)^{-4}\right),
$$

if $V$ satisfies (1.8), and we have used the bounds (2.1),(2.2) and (2.3) in the second case. Hence we can rewrite the above inequality in the form

$$
\begin{gathered}
\mu^{\prime} F+\mu F^{\prime} \geq\left(E \mu^{\prime}+A(r)-C B(r)\right)\|u(r, \cdot)\|^{2}+\frac{\mu^{\prime}}{3}\left\|\mathcal{D}_{r} u(r, \cdot)\right\|^{2} \\
-\frac{3 h^{-2} \mu^{2}}{\mu^{\prime}}\left\|\mathcal{P}_{\varphi}^{ \pm}(h) u(r, \cdot)\right\|^{2}-\varepsilon h^{-1} \mu\left(\|u(r, \cdot)\|^{2}+\left\|\mathcal{D}_{r} u(r, \cdot)\right\|^{2}\right)
\end{gathered}
$$

with a suitable constant $C>0$. Now we use Lemma 2.3 to conclude that

$$
\begin{gathered}
\mu^{\prime} F+\mu F^{\prime} \geq \frac{E}{2} \mu^{\prime}\|u(r, \cdot)\|^{2}+\frac{\mu^{\prime}}{3}\left\|\mathcal{D}_{r} u(r, \cdot)\right\|^{2}-\frac{3 h^{-2} \mu^{2}}{\mu^{\prime}}\left\|\mathcal{P}_{\varphi}^{ \pm}(h) u(r, \cdot)\right\|^{2} \\
-\varepsilon h^{-1} \mu\left(\|u(r, \cdot)\|^{2}+\left\|\mathcal{D}_{r} u(r, \cdot)\right\|^{2}\right) .
\end{gathered}
$$

We integrate this inequality with respect to $r$ and use that $\mu(0)=0$. We have

Thus we obtain the estimate

$$
\int_{0}^{\infty}\left(\mu^{\prime} F+\mu F^{\prime}\right) d r=0 .
$$

$$
\begin{gathered}
\frac{E}{2} \int_{0}^{\infty} \mu^{\prime}\|u(r, \cdot)\|^{2} d r+\int_{0}^{\infty} \frac{\mu^{\prime}}{3}\left\|\mathcal{D}_{r} u(r, \cdot)\right\|^{2} d r \leq 3 h^{-2} \int_{0}^{\infty} \frac{\mu^{2}}{\mu^{\prime}}\left\|\mathcal{P}_{\varphi}^{ \pm}(h) u(r, \cdot)\right\|^{2} d r \\
+\varepsilon h^{-1} \int_{0}^{\infty} \mu\left(\|u(r, \cdot)\|^{2}+\left\|\mathcal{D}_{r} u(r, \cdot)\right\|^{2}\right) d r
\end{gathered}
$$

Using that $\mu=\mathcal{O}\left(a^{2}\right)$ together with (2.6) we get from (5.3)

$$
\begin{aligned}
& \int_{0}^{\infty}(r+1)^{-2 s}\left(\|u(r, \cdot)\|^{2}+\left\|\mathcal{D}_{r} u(r, \cdot)\right\|^{2}\right) d r \\
& \leq C a^{4} h^{-2} \int_{0}^{\infty}(r+1)^{2 s}\left\|\mathcal{P}_{\varphi}^{ \pm}(h) u(r, \cdot)\right\|^{2} d r \\
& +C \varepsilon h^{-1} a^{2} \int_{0}^{\infty}\left(\|u(r, \cdot)\|^{2}+\left\|\mathcal{D}_{r} u(r, \cdot)\right\|^{2}\right) d r
\end{aligned}
$$

with some constant $C>0$ independent of $h$ and $\varepsilon$. On the other hand, we have the identity

$$
\operatorname{Re} \int_{0}^{\infty}\left\langle 2 i \varphi^{\prime} \mathcal{D}_{r} u(r, \cdot), u(r, \cdot)\right\rangle d r=\int_{0}^{\infty} h \varphi^{\prime \prime}\|u(r, \cdot)\|^{2} d r
$$


and hence

$$
\begin{gathered}
\operatorname{Re} \int_{0}^{\infty}\left\langle\mathcal{P}_{\varphi}^{ \pm}(h) u(r, \cdot), u(r, \cdot)\right\rangle d r=\int_{0}^{\infty}\left\|\mathcal{D}_{r} u(r, \cdot)\right\|^{2} d r+\int_{0}^{\infty}\left\langle r^{-2} \Lambda_{w} u(r, \cdot), u(r, \cdot)\right\rangle d r \\
-\int_{0}^{\infty}\left(E+\varphi^{\prime 2}\right)\|u(r, \cdot)\|^{2} d r+\int_{0}^{\infty}\langle V u(r, \cdot), u(r, \cdot)\rangle d r \\
\geq \int_{0}^{\infty}\left\|\mathcal{D}_{r} u(r, \cdot)\right\|^{2} d r-\mathcal{O}\left(\tau^{2}\right) \int_{0}^{\infty}\|u(r, \cdot)\|^{2} d r .
\end{gathered}
$$

This implies

$$
\begin{gathered}
\varepsilon h^{-1} a^{2} \int_{0}^{\infty}\left\|\mathcal{D}_{r} u(r, \cdot)\right\|^{2} d r \leq \mathcal{O}\left(\tau^{2}\right) \varepsilon h^{-1} a^{2} \int_{0}^{\infty}\|u(r, \cdot)\|^{2} d r \\
+\gamma \int_{0}^{\infty}(r+1)^{-2 s}\|u(r, \cdot)\|^{2} d r+\gamma^{-1} h^{-2} a^{4} \int_{0}^{\infty}(r+1)^{2 s}\left\|\mathcal{P}_{\varphi}^{ \pm}(h) u(r, \cdot)\right\|^{2} d r
\end{gathered}
$$

for every $\gamma>0$. Taking $\gamma$ small enough, independent of $h, \tau$ and $a$, and combining the estimates (5.4) and (5.5), we get

$$
\begin{gathered}
\int_{0}^{\infty}(r+1)^{-2 s}\left(\|u(r, \cdot)\|^{2}+\left\|\mathcal{D}_{r} u(r, \cdot)\right\|^{2}\right) d r \\
\leq C a^{4} h^{-2} \int_{0}^{\infty}(r+1)^{2 s}\left\|\mathcal{P}_{\varphi}^{ \pm}(h) u(r, \cdot)\right\|^{2} d r \\
+C \varepsilon h^{-1} a^{2} \tau^{2} \int_{0}^{\infty}\|u(r, \cdot)\|^{2} d r
\end{gathered}
$$

with a new constant $C>0$ independent of $h$ and $\varepsilon$. Clearly, the estimate (5.6) implies (4.1).

The proof of Theorem 4.2 in the case when $d \geq 3$ goes very much like the proof of Theorem 4.1 above. The only difference in this case is that we have to integrate the function $F(r)$ from $r_{0}$ to $\infty$ and use that $F\left(r_{0}\right)=0$ by assumption. Thus, by Lemma 2.3 we conclude that the inequality (5.2) holds for all $r \geq r_{0}$.

In the case $d=2$ the operator $\Lambda_{w}$ is no longer non-negative. Instead, we will use that so is the operator $-\Delta_{w}$. Thus, it is easy to see that the above inequalities still hold with $V_{L}$ replaced by $V_{L}-h^{2}(2 r)^{-2}$. Since

$$
h^{2}\left(\mu(r)(2 r)^{-2}\right)^{\prime}=h^{2} \mu^{\prime}(r)(2 r)^{-2}-2^{-1} h^{2} r^{-3} \mu(r)>-h^{2} r^{-3} \mu(r),
$$

we can use Lemmas 2.4 and 2.5 instead of Lemma 2.3 to conclude that the inequality (5.2) remains valid for $r \geq r_{0}$ with $E / 2$ replaced by $E / 3$.

\section{REFERENCES}

[1] N. BuRQ, Décroissance de l'énergie locale de l'équation des ondes pour le problème extérieur et absence de résonance au voisinage du réel, Acta Math. 180 (1998), 1-29.

[2] N. BuRQ, Lower bounds for shape resonances widths of long-range Schrödinger operators, Amer. J. Math. 124 (2002), 677-735.

[3] F. Cardoso and G. Vodev, Uniform estimates of the resolvent of the Laplace-Beltrami operator on infinite volume Riemannian manifolds, Ann. Henri Poincaré 4 (2002), 673-691.

[4] J. Galkowski And J. Shapiro, Semiclassical resolvent bounds for weakly decaying potentials, preprint 2020.

[5] K. Datchev, Quantative limiting absorption principle in the semiclassical limit, Geom. Funct. Anal. 24 (2014), 740-747.

[6] K. Datchev and J. Shapiro, Semiclassical estimates for scattering on the real line, Commun. Math. Phys. 376 (2020), 2301-2308. 
[7] F. Klopp And M. Vogel, Semiclassical resolvent estimates for bounded potentials, Pure Appl. Analysis 1 (2019), 1-25.

[8] G. Lebeau and L. Robbiano, Contrôle exact de l'équation de la chaleur, Commun. Partial Diff. Equations 20 (1995), 335-356.

[9] J. Shapiro, Local energy decay for Lipschitz wavespeeds, Commun. Partial Diff. Equations 43 (2018), 839-858.

[10] J. Shapiro, Semiclassical resolvent bounds in dimension two, Proc. Amer. Math. Soc. 147 (2019), 1999-2008.

[11] J. ShapiRo, Semiclassical resolvent bound for compactly supported $L^{\infty}$ potentials, J. Spectral Theory 10 (2020), 651-672.

[12] G. Vodev, Semiclassical resolvent estimates for short-range $L^{\infty}$ potentials, Pure Appl. Analysis 1 (2019), 207-214.

[13] G. Vodev, Semiclassical resolvent estimates for short-range $L^{\infty}$ potentials. II, Asymptotic Analysis 118 (2020), 297-312.

[14] G. Vodev, Semiclassical resolvent estimates for $L^{\infty}$ potentials on Riemannian manifolds, Ann. Henri Poincaré 21 (2020), 437-459.

[15] G. VodEv, Improved resolvent bounds for radial potentials, preprint 2020.

Université de Nantes, Laboratoire de Mathématiques Jean Leray, 2 rue de la Houssinière, BP 92208, 44322 Nantes Cedex 03, France

E-mail address: Georgi.Vodev@univ-nantes.fr 